\title{
X-ray crystallographic study of copper(II), cobalt(II) and silver(I) complexes of ligands containing two oxine subunits linked by xylylene spacer groups.
}

\author{
Muna R. A. Al-Mandhary ${ }^{a}$ and Peter J. Steel ${ }^{b^{*}}$ \\ ${ }^{a}$ College of Science, Sultan Qaboos University, PO Box 36, Al-Khod 123, Sultanate of Oman \\ ${ }^{b}$ Department of Chemistry, University of Canterbury, Christchurch, New Zealand
}

\begin{abstract}
X-Ray crystal structures are reported for six transition metal complexes of the ligands 1,2-bis(8-quinolyloxymethyl)benzene (3) and 1,3-bis(8-quinolyloxymethyl)benzene (4). In three mononuclear copper(II) and cobalt(II) complexes ligand $\mathbf{3}$ acts as a chelating ligand, with either N,O,N'-tridentate or N,O,O',N'-tetradentate coordination. A novel binuclear silver(I) complex of $\mathbf{3}$ is shown to be stabilised by a number of weak metal-ligand contacts and multiple intramolecular $\pi-\pi$ stacking interactions. In two binuclear copper(II) nitrate complexes of $\mathbf{4}$, the ligand adopts very different bridging modes of coordination.
\end{abstract}

Keywords: N,O ligands / Copper / Cobalt / Silver / Bridging ligands

\footnotetext{
* Corresponding author. Tel.: +64-3-3642432; fax: +64-3-3642110;

e-mail: p.steel@chem.canterbury.ac.nz.
} 
Introduction

Studies of combinations of organic ligands and metal ion precursors for the controlled self-assembly of metallosupramolecular species have been the subject of a number of recent reviews. ${ }^{[1]}$ Whereas rigid components are most commonly used for the rational construction of various molecular architectures, we have adopted the different approach of using flexible building blocks in order to gain access to topologies not accessible from more rigid precursors. In this context, for some years ${ }^{[2]}$ we have been synthesising a class of ligands represented by the general structure 1 (Scheme 1). Each of these ligands consists of a number of heterocyclic donor groups linked by flexible spacers $(\mathrm{X})$ to a central arene core. This simple representation disguises the complexity of a large library of compounds that we are currently investigating. By systematically varying the number of arms, the specific heterocycle, the nature of the linking groups and the type of arene core, we are preparing ligands with a diverse range of properties.

[Scheme 1 here]

We have found xylylene derivatives to be particularly useful components for the construction of various interesting new supramolecular architectures. For example, we have prepared new ligands consisting of ortho, meta and para xylylene groups connected to pyrazolyl, ${ }^{[3]}$ 2-pyridyloxy, ${ }^{[4]}$ 2-pyridylsulfanyl ${ }^{[5]}$ and benzotriazolyl ${ }^{[6]}$ moieties and used these in reactions with various metals to produce metallosupramolecular species of diverse dimensionalities. In such compounds, additional stability can be imparted to the metal complexes by incorporating chelating binding domains into the ligand design. Although bridging ligands containing multiple 2,2'-bipyridine binding sites are by far the most well studied, ${ }^{[7]}$ we, ${ }^{[8]}$ and others, ${ }^{[9]}$ have found the 3-(2-pyridyl)pyrazol-1-yl substituent to be a useful N,N'-chelating structural subunit and have attached this moiety to xylylene spacers.

Recently, we have embarked on the synthesis of a range of ligands that contain N,Ochelating moieties and have chosen 8-hydroxyquinoline (oxine) (2) as a common starting material. Although oxine itself has a long history as a versatile bidentate ligand, ${ }^{[10]}$ it has not commonly been incorporated into larger multidentate ligands. Recently, Albrecht and coworkers have reported the use of a number of ligands containing two oxine groups linked 
through the carbon atoms of the quinoline ring to prepare various complexes. ${ }^{[11]}$ However, our approach has been to join the oxine subunits via the oxygen atoms. ${ }^{[12,13]}$

We herein describe the syntheses and X-ray crystal structures of a number of transition metal complexes of ligands $\mathbf{3}$ and $\mathbf{4}$, which consist of two oxine groups attached to ortho- and meta-xylylene cores, respectively. Recently, we have communicated the intriguing ability of 4 to form a compact trinuclear circular helicate upon reaction with silver triflate. ${ }^{[14]}$ 


\section{Results and Discussion}

The ligands, 1,2-bis(8-quinolyloxymethyl)benzene (3) and 1,3-bis(8quinolyloxymethyl)benzene (4), are known compounds that were prepared by the literature procedures. ${ }^{[15,16]}$ We also prepared the corresponding para-isomer, ${ }^{[16]}$ but were unable to form any crystalline complexes from it. Related podands, including 3, have previously been shown to form stable complexes with alkali and alkaline earth metals. ${ }^{[17]}$ Ligands $\mathbf{3}$ and $\mathbf{4}$ were reacted with a variety of transition metal salts under various reaction conditions and stoichiometric ratios. Six such reactions produced crystalline products suitable for X-ray crystal structure analysis, the structures of which we now describe. These two ligands might be expected to adopt a variety of coordination modes depending on which of the donor atoms are involved. The ligands could act as bidentate N,N'-donors in chelating or bridging modes, or as tetradentate ligands with involvement of the weaker ether oxygen donors.

Reactions of 3 with copper(II) chloride, copper(II) nitrate and cobalt(II) bromide all furnished crystalline products in good yields. Each of these crystallizes in the triclinic space group P-1 and is a mononuclear complex with 1:1 L:MX ${ }_{2}$ stoichiometry. Figures 1-3 show perspective views, with selected interatomic distances and angles, of the three structures in similar orientations. Although the three structures appear very similar, there are some intriguing, subtle differences. In all three structures the ligand $\mathbf{3}$ adopts a very similar conformation, wherein the plane of each quinoline ring is almost orthogonal to the plane of the attached xylylene group (range of angles between planes $=86.3-88.8^{\circ}$ ). However, the structures differ in the extent of interaction of the ether oxygens with the metal centre. This, in turn, is reflected in different bonding geometries at the metal.

[Figures 1-3 here]

In the copper(II) chloride complex (5) the metal has approximately square pyramidal five-coordinate geometry, as defined by a $\tau$ value of $0.19 .^{[18]}$ In this arrangement the atoms N11, N21, O2 and Cl1 define the basal plane and Cl2 occupies the apical position and, accordingly, has a longer bond length to the copper. This geometry allows for a weak interaction [2.466(2) $\AA$ ] between the metal and the formally non-coordinated ether oxygen O1 (shown as a dashed line in Figure 1). However, in the copper(II) nitrate complex (6) the 
geometry is intermediate between square pyramidal and trigonal bipyramidal, with a $\tau$ value of 0.47 . Once again, this geometry permits a weak interaction [2.457(3) $\AA$ ] between the copper and $\mathrm{O} 2$.

In contrast, the greater requirement for six-coordination of cobalt results in complex 7 having distorted octahedral geometry with the ligand $\mathbf{3}$ employing all of its four donor atoms. This results in two N,O-five-membered chelate rings being fused to a central O,O'-sevenmembered chelate ring. The distortions from idealised octahedral geometry result from the chelate ring bite angles (each $c a 73^{\circ}$ ) and the Br1-Co-Br2 bond angle [108.09(2) ${ }^{\circ}$. Thus, in these three complexes the ligand $\mathbf{3}$ acts as a chelating ligand with either N,O,N'-tridentate or N,O,O',N'-tetradentate coordination.

Previously, ${ }^{[14]}$ we have described the reaction of $\mathbf{4}$ with silver triflate to produce a novel trinuclear circular helicate. Analogous reaction of $\mathbf{3}$ with silver triflate produced a very different result. The crystalline product (8) obtained from this reaction crystallizes in the monoclinic space group $\mathrm{P} 2{ }_{1} / \mathrm{c}$, with the asymmetric unit comprised of a $\mathrm{M}_{2} \mathrm{~L}_{3}$ unit, two triflate counterions and a diethyl ether solvate molecule. Figure 4 show a perspective view of the binuclear dication, along with selected interatomic distances and angles. The nearly symmetrical structure consists of a central molecule of $\mathbf{3}$ that bridges two silver atoms, each of which is further coordinated to another molecule of $\mathbf{3}$. The structure of $\mathbf{8}$ is interesting for a number of reasons. Each silver atom is coordinated to two quinoline nitrogens with near linear geometry. However, the bond lengths (>2.2 $\AA$ ) are somewhat long for two-coordinate silver. ${ }^{[19]}$ The reason for this is the involvement of other donors with the $\mathrm{d}^{10}$ silver centres.

[Figure 4 here]

The oxygen atoms attached to the externally coordinated quinoline rings are involved in weak chelation ( ca $2.58 \AA$ ) with the silver atoms (hollow bonds in Figure 4). A notable feature of the structure is the unusual and symmetrical conformations of three molecules of $\mathbf{3}$ in this complex. We believe that each of these ligands contorts itself into the observed conformations in order to bring the remaining six potential donors into proximity with the silver atoms. Although each of these interactions (dashed bonds in Figure 4) is undoubtedly weak (ca $2.7 \AA$ ), the combined forces of all six such interactions is likely to have a 
significantly stabilising influence. There is also a further stabilising factor operating in this complex, viz intramolecular $\pi-\pi$ stacking interactions between the various aromatic ring systems. Specifically, the central benzene ring of the bridging ligand is stacked between the two chelating quinolines. Additionally, the two quinoline rings of the central bridging ligand are stacked on top of one another and between the benzene rings of the outer two ligands. All of these interactions involve coplanar rings (angles between planes $<5^{\circ}$ ) separated by ca 3.4 Å. Once again, although these are weak interactions, the combined influence of all five stacking interactions is likely to be significant.

Having demonstrated that ligand $\mathbf{3}$ is capable of acting as both a chelating and bridging ligand, we turned our attention to the meta isomer $\mathbf{4}$, where a chelating mode of coordination would appear less likely. Ligand 4 was reacted with copper(II) nitrate in a variety of different solvents and in two cases this provided crystalline products, which were shown to be structurally very different. Reaction in methanol produced complex $\mathbf{9}$, which crystallizes in the monoclinic space group $\mathrm{P} 2_{1} / \mathrm{n}$. Figure 5 shows a perspective view, with selected interatomic distances and angles, of the structure of $\mathbf{9}$.

[Figure 5 here]

The complex consists of a binuclear copper pair bridged by two methoxide anions and ligand 4. Monodentate nitrate anions complete the coordination environment of the two square planar copper atoms. Once again the bridging ligand $\mathbf{4}$ adopts an unusual conformation, which we believe occurs in order to bring the non-coordinated ether oxygens into the coordination sphere of the copper atoms (dashed bonds in Figure 5). Relatively few binuclear methoxide bridged copper complexes of this type are known. ${ }^{[20]}$ The Cu1-Cu2

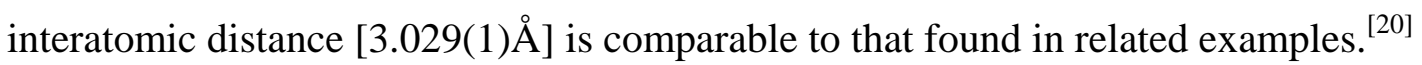

In contrast to this result, reaction of $\mathbf{4}$ with copper(II) nitrate in acetonitrile led to a complex (10) with very different architecture. It crystallizes in the triclinic space group P-1 and has the structure show in Figure 6. In this complex the ligand $\mathbf{4}$ acts as a more conventional bridging ligand separating two copper atoms at a distance of 10.526(1) Å. The copper atoms have square pyramidal geometry ( $\tau$ values 0.08 and 0.02 for $\mathrm{Cu} 1$ and $\mathrm{Cu} 2$, respectively) and are further coordinated to two monodentate nitrates, a water molecule and, 
with considerably longer bonds, to ether oxygens in the apical positions. Surprisingly, a search of the Cambridge Crystallographic Database revealed that this is an unprecedented geometry for a copper atom with this donor set.

[Figure 6 here]

Thus, the ligand $\mathbf{4}$ acts as a bridging ligand in each of these two copper complexes, but, in doing so, produces binuclear complexes with very different topologies. As mentioned above, we have previously reported a novel trinuclear circular helicate resulting from reaction of $\mathbf{4}$ with silver triflate. We have since reacted $\mathbf{4}$ with silver nitrate and found that this leads to a similar circular helicate, but the X-ray crystal structure of which we were unable to refine to a level that we consider to be acceptable (currently $\mathrm{R}_{1}=6.5 \%$ ). Nevertheless, this result shows that the formation of such circular helicates occurs independently of the counterion, thereby suggesting that the self-assembly process does not involve anion templation. ${ }^{[21]}$

\section{Conclusion}

In this study, we have shown that ligands comprised of two oxine subunits separated by xylylene spacer groups represent useful substrates for the construction of a diverse range of interesting new complexes of varying nuclearity. These ligands have been shown to be capable of both chelating and bridging modes of coordination. Presently, we are extending this design concept to related ligands containing a greater number of oxine substructures, thereby providing access to more complex multinuclear species.

\section{Acknowledgement}

We thank the Royal Society of New Zealand Marsden Fund for financial support and Sultan Qaboos University for sabbatical leave to M. Al-Mandhary. We also thank Chris Fitchett and John Cannon for important contributions to the crystallography. 


\section{Experimental}

Ligands $3^{[15]}$ and $4^{[16]}$ were prepared by literature procedures. Solvents were purified according to literature procedures. Other reagents were obtained from commercial sources and used as supplied. Melting points were determined using an Electrothermal melting point apparatus and are uncorrected. Elemental analyses were performed by the Campbell Microanalytical laboratory at the University of Otago.

Complex 5. $\mathrm{CuCl}_{2} .2 \mathrm{H}_{2} \mathrm{O}(8.7 \mathrm{mg}, 0.051 \mathrm{mmol})$ dissolved in $\mathrm{MeCN}(1 \mathrm{~mL})$ was layered onto a solution of ligand 3 (20 mg, $0.051 \mathrm{mmol}$ ) in $\mathrm{CH}_{2} \mathrm{Cl}_{2}$ (1 $\left.1 \mathrm{~mL}\right)$ to produce orange crystals suitable for X-ray analysis. M.p. $168^{\circ} \mathrm{C}$. Yield $21 \mathrm{mg}$ (78\%). Elemental analysis: $\mathrm{C}_{26} \mathrm{H}_{20} \mathrm{~N}_{2} \mathrm{O}_{2} \mathrm{Cl}_{2} \mathrm{Cu}$ (526.90) requires $\mathrm{C}, 59.27 ; \mathrm{H}, 3.83 ; \mathrm{N}, 5.32$; found $\mathrm{C}, 59.25 ; \mathrm{H}, 4.00 ; \mathrm{N}$, 5.41 .

Complex 6. $\mathrm{Cu}\left(\mathrm{NO}_{3}\right)_{2} .3 \mathrm{H}_{2} \mathrm{O}(12 \mathrm{mg}, 0.050 \mathrm{mmol})$ dissolved in $\mathrm{MeCN}(1 \mathrm{~mL})$ was layered onto a solution of ligand 3 (20 mg, $0.051 \mathrm{mmol})$ in $\mathrm{CH}_{2} \mathrm{Cl}_{2}(1 \mathrm{~mL})$ to produce yellow crystals suitable for X-ray analysis. M.p. 140-142 ${ }^{\circ} \mathrm{C}$. Yield $24 \mathrm{mg}$ (71\%). Analysis: $\mathrm{C}_{26} \mathrm{H}_{20} \mathrm{~N}_{4} \mathrm{O}_{8} \mathrm{Cu} . \mathrm{CH}_{2} \mathrm{Cl}_{2}$ (664.94) requires C, 48.77; H, 3.33; N, 8.43; found C, 49.13; H, 3.13; N, 8.41.

Complex 7. $\mathrm{CoBr}_{2}$ (22 mg, $0.10 \mathrm{mmol}$ ) dissolved in $\mathrm{MeOH}(1 \mathrm{~mL})$ was layered onto a solution of ligand 3 (20 mg, $0.051 \mathrm{mmol})$ in $\mathrm{CH}_{2} \mathrm{Cl}_{2}(1 \mathrm{~mL})$ to give purple crystals suitable for X-ray analysis. M.p. $240^{\circ} \mathrm{C}$ (decomp). Yield $22 \mathrm{mg}$ (71\%). Analysis: $\mathrm{C}_{26} \mathrm{H}_{20} \mathrm{~N}_{2} \mathrm{O}_{2} \mathrm{CoBr}_{2}$ (611.19) requires C, 51.09; H, 3.30; N, 4.58; found C, 51.17; H, 3.19; N, 4.67.

Complex 8. AgOTf (8.7 mg, $0.034 \mathrm{mmol})$ dissolved in $\mathrm{MeCN}(1 \mathrm{~mL})$ was added to a solution of ligand 3 (20 mg, $0.051 \mathrm{mmol})$ dissolved in MeCN (1mL). Diethyl ether (1mL) was then added to this solution. Slow evaporation of this mixture provided colourless crystals suitable for X-ray analysis. M.p. 226-229 ${ }^{\circ} \mathrm{C}$. Yield $20 \mathrm{mg}$ (70\%). Analysis:

$\mathrm{C}_{80} \mathrm{H}_{60} \mathrm{~N}_{6} \mathrm{O}_{12} \mathrm{~F}_{6} \mathrm{~S}_{2} \mathrm{Ag}_{2}$ (1691.22) requires C, 56.81; H, 3.58; N, 4.97; found C, 56.63; H, 3.54; $\mathrm{N}, 4.91$.

Complex 9. $\mathrm{Cu}\left(\mathrm{NO}_{3}\right)_{2} .3 \mathrm{H}_{2} \mathrm{O}$ (25.6 mg, $0.102 \mathrm{mmol}$ ) dissolved in $\mathrm{MeOH}(1 \mathrm{~mL})$ was layered onto a solution of ligand 4 (20 mg, $0.051 \mathrm{mmol})$ in $\mathrm{CH}_{2} \mathrm{Cl}_{2}(1 \mathrm{~mL})$ to give dark green 
crystals suitable for X-ray analysis. M.p. $205^{\circ} \mathrm{C}$ (decomp). Yield $11 \mathrm{mg}$ (31\%). Analysis: $\mathrm{C}_{28} \mathrm{H}_{26} \mathrm{~N}_{4} \mathrm{O}_{10} \mathrm{Cu}_{2}$ (705.62) requires $\mathrm{C}$, 47.66; $\mathrm{H}, 3.71 ; \mathrm{N}, 7.94$; found $\mathrm{C}$, 47.69; $\mathrm{H}, 3.85 ; \mathrm{N}$, 7.91.

Complex 10. $\mathrm{Cu}\left(\mathrm{NO}_{3}\right)_{2} .3 \mathrm{H}_{2} \mathrm{O}(12 \mathrm{mg}, 0.051 \mathrm{mmol})$ dissolved in $\mathrm{MeCN}(1 \mathrm{~mL})$ was layered onto a solution of ligand 4 (10 mg, $0.025 \mathrm{mmol})$ in $\mathrm{CH}_{2} \mathrm{Cl}_{2}(1 \mathrm{~mL})$ to give a green solution. Diffusion of diethyl ether into this solution gave green crystals suitable for X-ray analysis. M.p. $118{ }^{\circ} \mathrm{C}$ (decomp). Yield $14 \mathrm{mg}$ (58\%). Analysis: $\mathrm{C}_{32} \mathrm{H}_{37} \mathrm{~N}_{7} \mathrm{O}_{17} \mathrm{Cu}_{2} \cdot{ }^{1 / 2}\left(\mathrm{CH}_{2} \mathrm{Cl}_{2}\right)$ (961.23) requires C, 41.61; H, 3.98; N, 10.21; found C, 41.27; H, 3.68; N, 10.34.

\section{X-Ray Crystallography}

The crystal data and details of the data collections and refinements for the six structures are listed in Table 1. Measurements were made with a SMART CCD area detector using graphite-monochromatized Mo $\mathrm{K} \alpha(\lambda=0.71073 \AA)$ radiation. The structures were solved by direct methods using SHELXS ${ }^{[22]}$ and refined on $\mathrm{F}^{2}$ using all data by full-matrix least-squares procedures with SHELXL-97. ${ }^{[23]}$ All non-hydrogen atoms were refined with anisotropic displacement parameters. Hydrogen atoms were included in calculated positions with isotropic displacement parameters 1.2 times the isotropic equivalent of their carrier atoms. The functions minimised were $\Sigma \mathrm{w}\left(\mathrm{F}_{\mathrm{o}}^{2}-\mathrm{F}_{\mathrm{c}}{ }^{2}\right)$, with $\mathrm{w}=\left[\sigma^{2}\left(\mathrm{~F}_{\mathrm{o}}^{2}\right)+\mathrm{aP}^{2}+\mathrm{bP}\right]^{-1}$, where $\mathrm{P}=\left[\max \left(\mathrm{F}_{\mathrm{O}}\right)^{2}+2 \mathrm{~F}_{\mathrm{C}}^{2}\right] / 3$. Full tables of atom coordinates, thermal parameters, and bond lengths and angles have been deposited at the Cambridge Crystallographic Data Centre. CCDC 194259-194264 contains the supplementary crystallographic data for this paper. These data can be obtained free of charge at www.ccdc.cam.ac.uk/conts/retrieving.html [or from the Cambridge Crystallographic Data Centre, 12 Union Road, Cambridge CB2 1EZ, UK; fax: (internat.) + 44-1223/336-033; Email: deposit@ccdc.cam.ac.uk]. 


\section{References}

1 For recent reviews, see: ${ }^{[1 \mathrm{a}]}$ S. Leininger, B. Olenyuk, P. J. Stang, Chem. Rev., 2000, 100, 853-908. ${ }^{[1 b]}$ G. F. Swiegers, T. J. Malefetse, Chem. Rev., 2000, 100, 3483-3538. ${ }^{[1 c]}$ B. Moulton, M. J. Zaworotko, Chem. Rev., 2001, 101, 1629-1658. ${ }^{[1 \mathrm{~d}]}$ M. Fujita, K. Umemoto, M. Yoshizawa, N. Fujita, T. Kusukawa, K. Biradha, Chem. Commun., 2001, 509-518. ${ }^{[1 \mathrm{e}]}$ D. W. Johnson, K. N. Raymond, Supramol. Chem., 2001, 13, 639-659. ${ }^{[1 f]}$ B. J. Holliday, C. A. Mirkin, Angew. Chem. Int. Ed., 2001, 40, 2022-2043. ${ }^{[1 \mathrm{~g}]}$ G. F. Swiegers, T. J. Malefetse, Coord. Chem. Rev., 2002, 225, 91-121.

2 C. M. Hartshorn, P. J. Steel, Inorg. Chem., 1996, 35, 6902-6903.

$3 \quad{ }^{[3 a]}$ C. M. Hartshorn, P. J. Steel, Aust. J. Chem., 1995, 48, 1587-1599. ${ }^{[3 b]}$ C. M. Hartshorn, P. J. Steel, Organometallics, 1998, 17, 3487-3496.

4 C. M. Hartshorn, P. J. Steel, J. Chem. Soc., Dalton Trans., 1998, 3927-3934.

5 C. M. Hartshorn, P. J. Steel, J. Chem. Soc., Dalton Trans., 1998, 3935-3940.

6 B. J. O’Keefe, P. J. Steel, Inorg. Chem. Commun., 2000, 3, 473-475.

7 C. Kaes, A. Katz, M. W. Hosseini, Chem. Rev., 2000, 100, 3553-3590.

8 C. M. Hartshorn, P. J. Steel, Inorg. Chem. Commun., 2000, 3, 476-481.

9 M. D. Ward, J. A. McCleverty, J. C. Jeffery, Coord. Chem. Rev., 2001, 222, 251-272.

10 J. P. Phillips, Chem. Rev., 1956, 56, 271-297.

$11{ }^{[11 \mathrm{a}]}$ M. Albrecht, O. Blau, Synthesis, 1997, 213-216. ${ }^{[11 \mathrm{~b}]}$ M. Albrecht, O. Blau, Chem. Commun., 1997, 345-346. ${ }^{[11 c]}$ M. Albrecht, O. Blau, R. Fröhlich, Chem. Eur. J., 1999, 5, 48-56. ${ }^{[11 \mathrm{~d}]}$ M. Albrecht, O. Blau, J. Zauner, Eur. J. Org. Chem., 1999, 3165-3169. ${ }^{[11 e]}$ M. Albrecht, O. Blau, E. Wegelius, K. Rissanen, New J. Chem., 1999, 23, 667-668.

12 M. R. A. Al-Mandhary, P. J. Steel, Aust. J. Chem., 2002, 55, 705-708.

13 M. R. A. Al-Mandhary, P. J. Steel, Inorg. Chim. Acta, 2003, 351. 7-11.

14 M. R. A. Al-Mandhary, P. J. Steel, Inorg. Chem. Commun., 2002, 5, 954-957.

15 K. Hiratani, K. Taguchi, H. Sugihara, T. Okaida, Chem. Lett., 1986, 197-200.

16 B. Tümmler, G. Maass, F. Vögtle, H. Sieger, U. Heimann, E. Weber, J. Am. Chem. Soc., 1979, 101, 2588-2598.

17 F. Vögtle, E. Weber, Angew. Chem. Int. Ed. Engl., 1979, 18, 753-776.

18 A. W. Addison, T. Nageswara Rao, J. Reedijk, J. van Rijn, G. C. Verschoor, J. Chem. Soc., Dalton. Trans., 1984, 1349-1356. 
19 M. Munakata, L. P. Wu, T. Kuroda-Sowa, Adv. Inorg. Chem., 1998, 46, 173-303.

$20{ }^{[19 a]}$ V. K. Voronkova, L. V. Mosina, Y. V. Yablokov, M. A. Yampolskaya, G. S. Matuzenko, Y. A. Simonov, B. Y. Kuyavskaya, N. V. Gerbeleu, V. K. Belskii, Zh.

Strukt. Khim., 1986, 27, 69-79. ${ }^{[19 b]}$ S. A. Komaei, G. A. van Albada, I. Mutikainen, U. Turpeinen, J. Reedijk, Eur. J. Inorg. Chem., 1998, 1577-1579. ${ }^{[19 c]}$ D. M. L. Goodgame, I. Hussain, A. J. P. White, D. J. Williams, J. Chem. Soc., Dalton Trans., 1999, 28992900. ${ }^{[19 d]}$ A. M. Guidote, K. Ando, K. Terada, Y. Kurusu, H. Nagao, Y. Matsuyama, Inorg. Chim. Acta, 2001, 324, 203-211.

$21{ }^{[20 \mathrm{a}]}$ M. Albrecht, Chem. Rev., 2001, 101, 3457-3497. ${ }^{[20 b]}$ R. Vilar, Angew. Chem. Int. Ed., 2003, 42, 1460-1477.

22 G. M. Sheldrick, Acta Crystallogr., Sect A, 1990, 46, 467-473.

23 G. M. Sheldrick, SHELXL-97, University of Göttingen, 1997. 
Scheme 1.

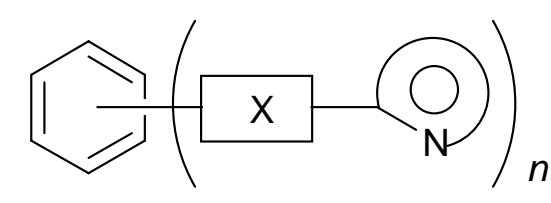

(1)

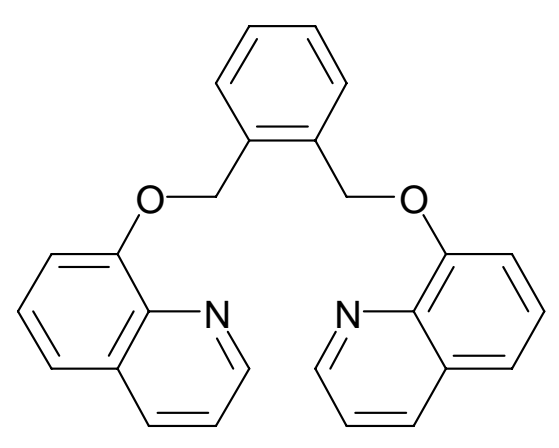

(3)<smiles>Oc1cccc2cccnc12</smiles>

(2)<smiles>c1cc(COc2cccc3cccnc23)cc(COc2cccc3cccnc23)c1</smiles>

(4) 
Figure Captions:

Fig. 1 Perspective view of the X-ray crystal structure of 5. Selected interatomic distances ( $\AA$ ) and angles ( ${ }^{\circ}$ ): Cu1-N11 2.025(2), Cu1-N21 2.024(2), Cu1-O1 2.466(2), Cu1-O2 2.261(2), Cu1-Cl1 2.2856(12), Cu1-Cl2 2.3688(12), N21-Cu1-N11 167.28(8), N21-Cu1-O2 74.95(8), N11-Cu1-O2 95.87(8), N21-Cu1-Cl1 95.18(7), N11-Cu1-Cl1 89.82(7), O2-Cu1Cl1 155.85(5), N21-Cu1-Cl2 92.18(6), N11-Cu1-Cl2 96.10(6), O1-Cu1-Cl2 149.80(5), O2Cu1-Cl2 86.59(5), Cl1-Cu1-Cl2 116.19(3).

Fig. 2 Perspective view of the X-ray crystal structure of $\mathbf{6}$. The dichloromethane solvate molecule is not shown. Selected interatomic distances $(\AA)$ and angles $\left({ }^{\circ}\right)$ : Cu1-N11 1.983(3), Cu1-N21 1.998(3), Cu1-O1 2.162(3), Cu1-O2 2.457(3), Cu1-O21 2.184(4), Cu1-O11 2.217(3), N11-Cu1-N21 173.17(13), N11-Cu1-O1 77.81(11), N21-Cu1-O1 99.66(11), N11Cu1-O21 91.58(13), N21-Cu1-O21 86.94(12), O1-Cu1-O21 144.85(11), N11-Cu1-O11 88.03(12), N21-Cu1-O11 98.08(12), O1-Cu1-O11 84.32(10), O2-Cu1-O11 150.70(10), O21Cu1-O11 129.23(11).

Fig. 3 Perspective view of the X-ray crystal structure of 7. Selected interatomic distances ( $\AA$ ) and angles ( ${ }^{\circ}$ ): Co-N11 2.153(2), Co-N21 2.157(2), Co-O1 2.211(2), Co-O2 2.282(2), Co-Br2 2.490(1), Co-Br1 2.496(1), N11-Co-N21 169.93(8), N11-Co-O1 73.76(7), N21-CoO1 99.18(7), N11-Co-O2 97.68(8), N21-Co-O2 73.06(8), O1-Co-O2 72.54(7), N11-Co-Br2 92.36(6), N21-Co-Br2 94.86(6), O1-Co-Br2 90.15(5), O2-Co-Br2 156.49(5), N11-Co-Br1 94.97(6), N21-Co-Br1 89.45(6), O1-Co-Br1 159.18(5), O2-Co-Br1 92.22(5), Br2-Co-Br1 108.09(2).

Fig. 4 Perspective view of the X-ray crystal structure of $\mathbf{8}$. Hydrogen atoms, the triflate counterions and the diethyl ether solvate molecule are all omitted for clarity. Selected interatomic distances ( $\AA$ ) and angles $\left({ }^{\circ}\right)$ : Ag1-N41 2.213(2), Ag1-N31 2.229(2), Ag1-O2 2.595(2), Ag1-O1 2.714(2), Ag1-N11 2.714(2), Ag1-O3 2.656(2), Ag2-N61 2.230(2), Ag2N71 2.261(2), Ag2-O5 2.575(2), Ag2-O4 2.722(2), Ag2-N91 2.722(2), Ag2-O6 2.725(2), N41-Ag1-N31 163.74(8), N41-Ag1-O2 125.39(7), N31-Ag1-O2 66.56(7), N61-Ag2-N71 167.94(8), N61-Ag2-O5 122.13(8), N71-Ag2-O5 66.53(8). 
Fig. 5 Perspective view of the X-ray crystal structure of $\mathbf{9}$. Selected interatomic distances (Å) and angles ( ${ }^{\circ}$ ): Cu1-O7 1.928(3), Cu1-O8 1.935(3), Cu1-O11 1.996(4), Cu1-N11 2.032(4), Cu1-O1 2.451(3), Cu1-Cu2 3.029(1), Cu2-O8 1.912(3), Cu2-O7 1.946(3), Cu2O21 1.985(3), Cu2-N21 2.043(4), Cu2-O2 2.492(3), O7-Cu1-O8 76.56(14), O7-Cu1-O11 96.85(14), O8-Cu1-O11 172.70(14), O7-Cu1-N11 165.39(17), O8-Cu1-N11 95.02(16), O11Cu1-N11 92.08(16), O8-Cu2-O7 76.67(14), O8-Cu2-O21 168.07(14), O7-Cu2-O21 93.42(15), O8-Cu2-N21 94.69(15), O7-Cu2-N21 170.47(16), O21-Cu2-N21 94.66(16).

Fig. 6 Perspective view of the X-ray crystal structure of 10. Diethyl ether and acetonitrile solvate molecules are not shown. Selected interatomic distances $(\AA)$ and angles $\left({ }^{\circ}\right)$ :Cu1-O8 1.935(2), Cu1-N21 1.985(2), Cu1-O43 1.987(2), Cu1-O31 1.990(2), Cu1-O2 2.391(2), Cu2O7 1.937(2), Cu2-N11 1.975(2), Cu2-O11 1.987(2), Cu2-O21 2.010(2), Cu2-O1 2.395(2), O8-Cu1-N21 177.78(9), O8-Cu1-O43 87.87(9), N21-Cu1-O43 91.59(8), O8-Cu1-O31 86.74(9), N21-Cu1-O31 93.96(8), O43-Cu1-O31 172.91(7), O8-Cu1-O2 107.48(9), N21Cu1-O2 74.68(7), O43-Cu1-O2 90.45(7), O31-Cu1-O2 86.78(7), O7-Cu2-N11 175.81(10), O7-Cu2-O11 88.90(9), N11-Cu2-O11 94.01(9), O7-Cu2-O21 86.22(9), N11-Cu2-O21 90.98(9), O11-Cu2-O21 174.68(8), O7-Cu2-O1 102.09(9), N11-Cu2-O1 74.76(8), O11-Cu2O1 93.34(8), O21-Cu2-O1 89.74(8). 
Fig. 1 Perspective view of the X-ray crystal structure of 5. Selected interatomic distances (Å) and angles ( ${ }^{\circ}$ ): Cu1-N11 2.025(2), Cu1-N21 2.024(2), Cu1-O1 2.466(2), Cu1-O2 2.261(2), Cu1-Cl1 2.2856(12), Cu1-Cl2 2.3688(12), N21-Cu1-N11 167.28(8), N21-Cu1-O2 74.95(8), N11-Cu1-O2 95.87(8), N21-Cu1-Cl1 95.18(7), N11-Cu1-Cl1 89.82(7), O2-Cu1Cl1 155.85(5), N21-Cu1-Cl2 92.18(6), N11-Cu1-Cl2 96.10(6), O1-Cu1-Cl2 149.80(5), O2Cu1-Cl2 86.59(5), Cl1-Cu1-Cl2 116.19(3).

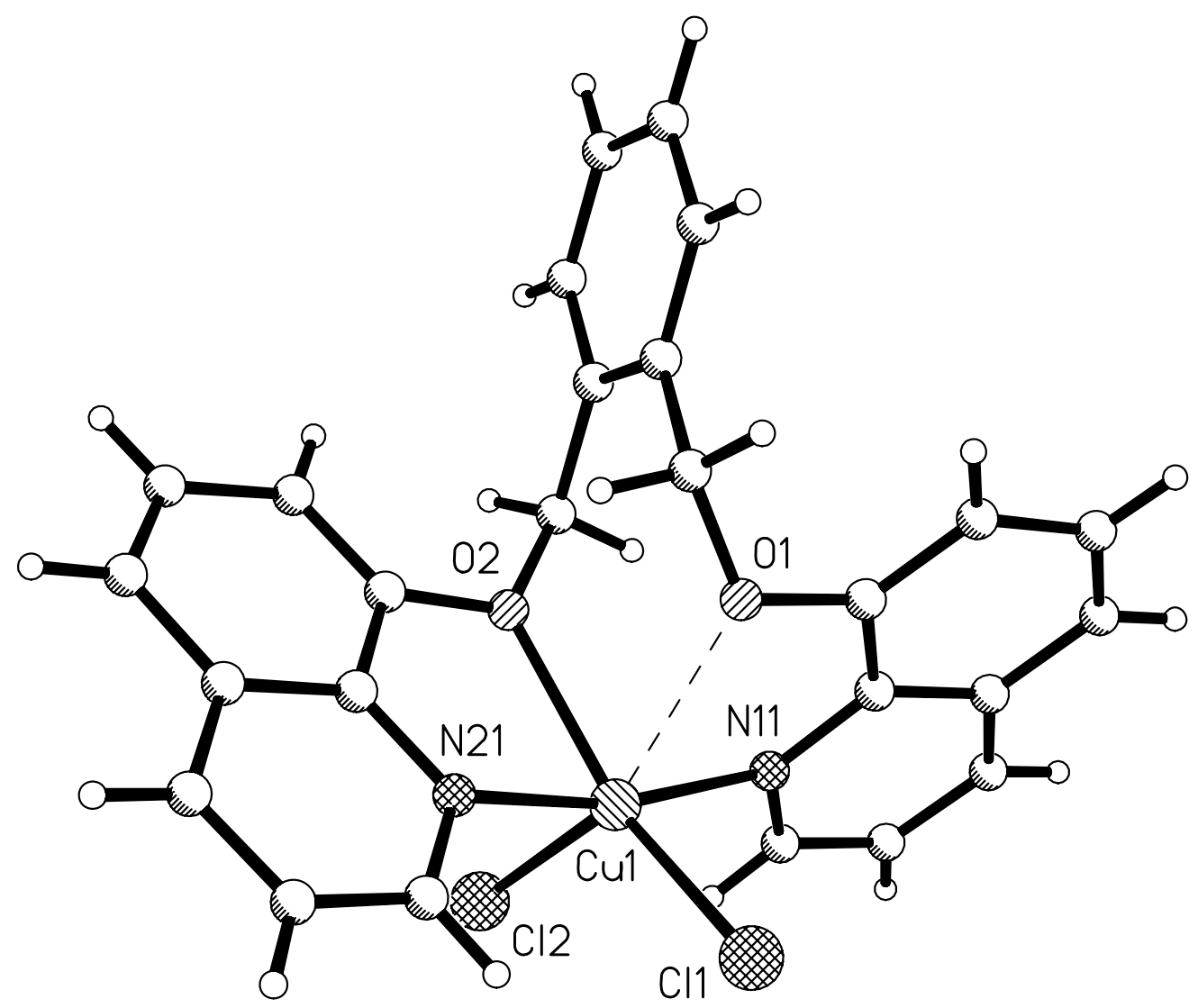


Fig. 2 Perspective view of the X-ray crystal structure of $\mathbf{6}$. The dichloromethane solvate molecule is not shown. Selected interatomic distances $(\AA)$ and angles $\left({ }^{\circ}\right)$ : Cu1-N11 1.983(3), Cu1-N21 1.998(3), Cu1-O1 2.162(3), Cu1-O2 2.457(3), Cu1-O21 2.184(4), Cu1-O11 2.217(3), N11-Cu1-N21 173.17(13), N11-Cu1-O1 77.81(11), N21-Cu1-O1 99.66(11), N11Cu1-O21 91.58(13), N21-Cu1-O21 86.94(12), O1-Cu1-O21 144.85(11), N11-Cu1-O11 88.03(12), N21-Cu1-O11 98.08(12), O1-Cu1-O11 84.32(10), O2-Cu1-O11 150.70(10), O21Cu1-O11 129.23(11).

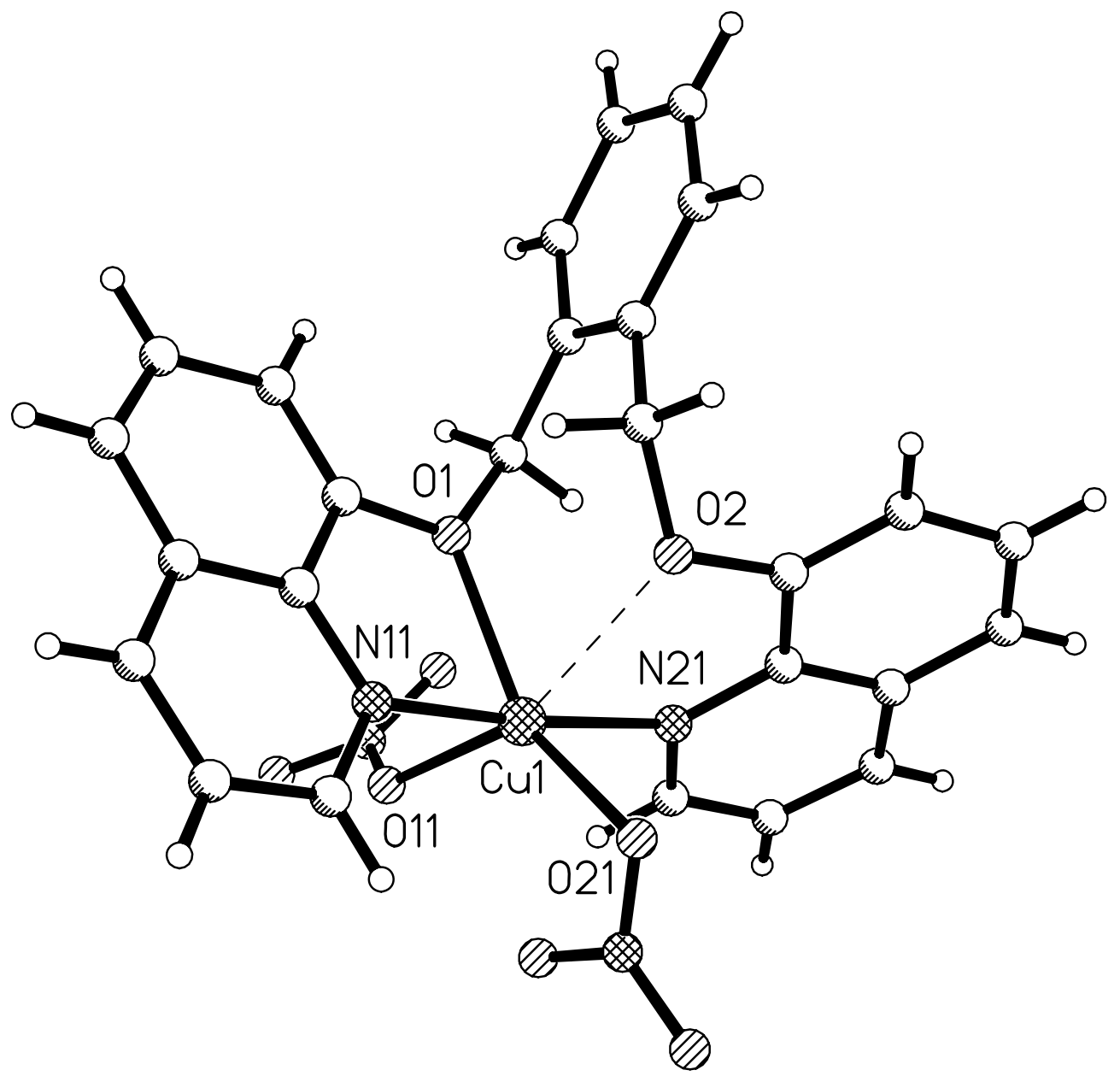


Fig. 3 Perspective view of the X-ray crystal structure of 7. Selected interatomic distances ( $\AA$ ) and angles $\left({ }^{\circ}\right)$ : Co-N11 2.153(2), Co-N21 2.157(2), Co-O1 2.211(2), Co-O2 2.282(2), Co-Br2 2.490(1), Co-Br1 2.496(1), N11-Co-N21 169.93(8), N11-Co-O1 73.76(7), N21-CoO1 99.18(7), N11-Co-O2 97.68(8), N21-Co-O2 73.06(8), O1-Co-O2 72.54(7), N11-Co-Br2 92.36(6), N21-Co-Br2 94.86(6), O1-Co-Br2 90.15(5), O2-Co-Br2 156.49(5), N11-Co-Br1 94.97(6), N21-Co-Br1 89.45(6), O1-Co-Br1 159.18(5), O2-Co-Br1 92.22(5), Br2-Co-Br1 108.09(2).

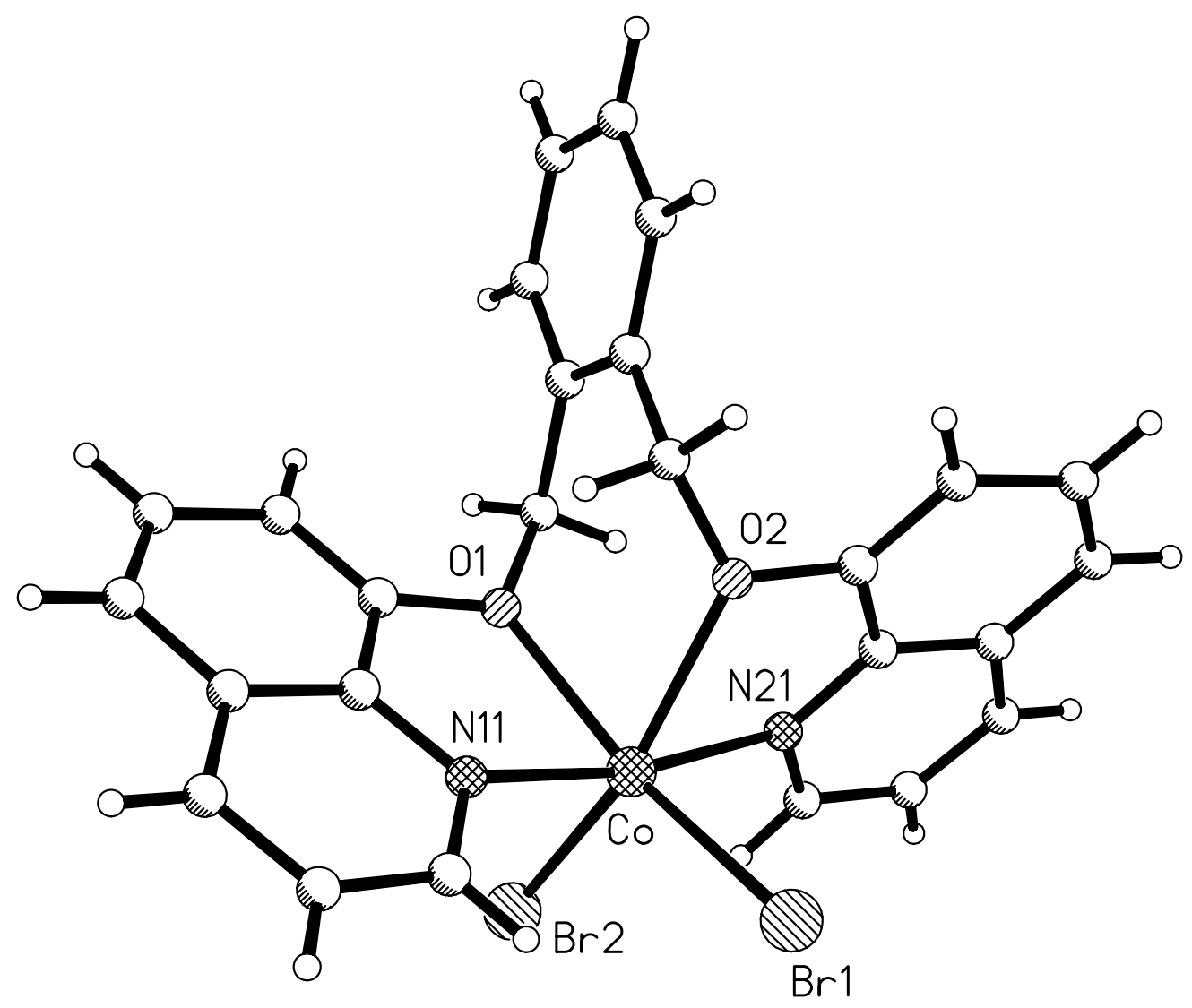


Fig. 4 Perspective view of the X-ray crystal structure of $\mathbf{8}$. Hydrogen atoms, the triflate counterions and the diethyl ether solvate molecule are all omitted for clarity. Selected interatomic distances $(\AA)$ and angles $\left({ }^{\circ}\right)$ : Ag1-N41 2.213(2), Ag1-N31 2.229(2), Ag1-O2 2.595(2), Ag1-O1 2.714(2), Ag1-N11 2.714(2), Ag1-O3 2.656(2), Ag2-N61 2.230(2), Ag2N71 2.261(2), Ag2-O5 2.575(2), Ag2-O4 2.722(2), Ag2-N91 2.722(2), Ag2-O6 2.725(2), N41-Ag1-N31 163.74(8), N41-Ag1-O2 125.39(7), N31-Ag1-O2 66.56(7), N61-Ag2-N71 167.94(8), N61-Ag2-O5 122.13(8), N71-Ag2-O5 66.53(8).

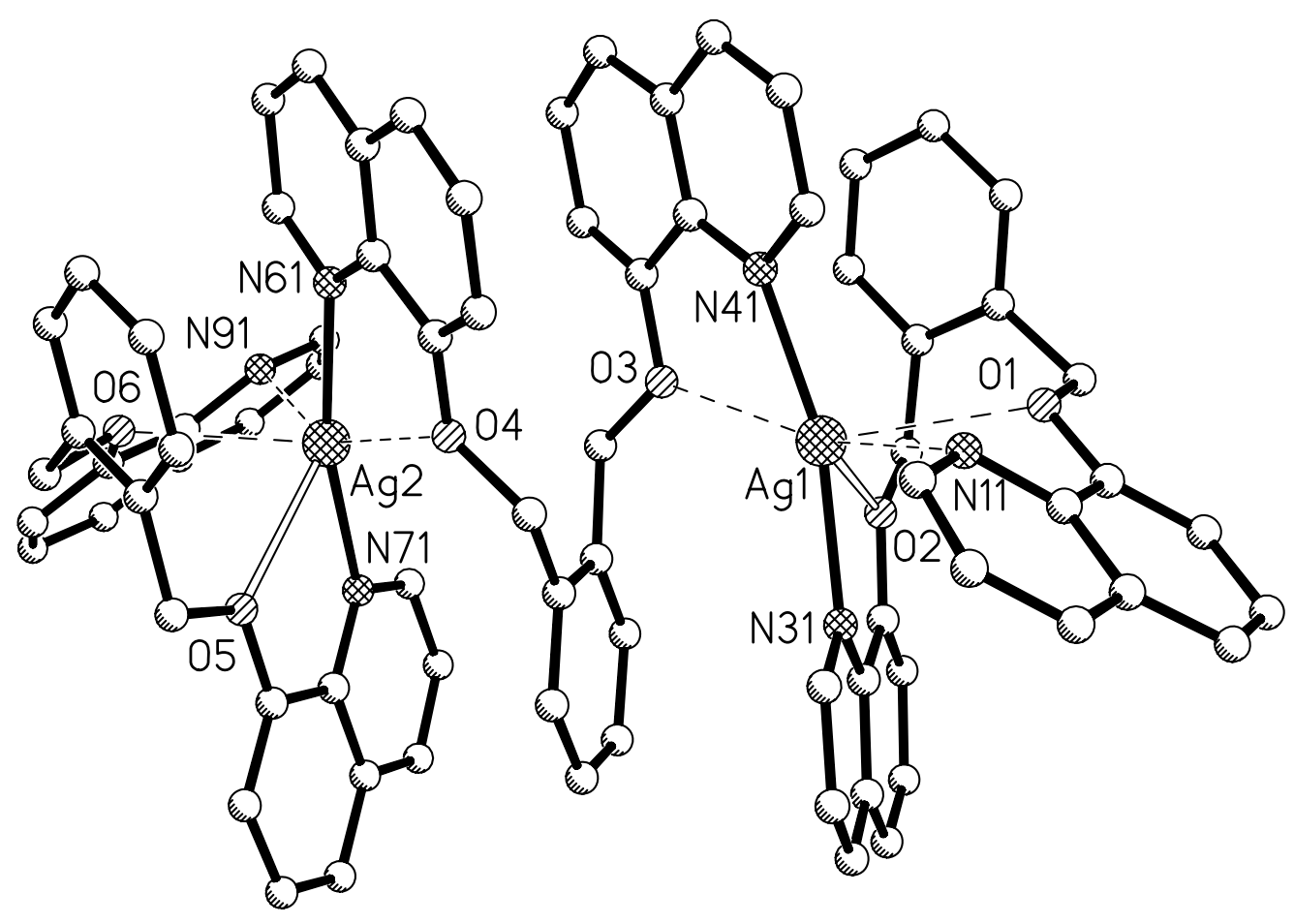


Fig. 5 Perspective view of the X-ray crystal structure of $\mathbf{9}$. Selected interatomic distances (Å) and angles ( $\left.{ }^{\circ}\right)$ : Cu1-O7 1.928(3), Cu1-O8 1.935(3), Cu1-O11 1.996(4), Cu1-N11 2.032(4), Cu1-O1 2.451(3), Cu1-Cu2 3.029(1), Cu2-O8 1.912(3), Cu2-O7 1.946(3), Cu2O21 1.985(3), Cu2-N21 2.043(4), Cu2-O2 2.492(3), O7-Cu1-O8 76.56(14), O7-Cu1-O11 96.85(14), O8-Cu1-O11 172.70(14), O7-Cu1-N11 165.39(17), O8-Cu1-N11 95.02(16), O11Cu1-N11 92.08(16), O8-Cu2-O7 76.67(14), O8-Cu2-O21 168.07(14), O7-Cu2-O21 93.42(15), O8-Cu2-N21 94.69(15), O7-Cu2-N21 170.47(16), O21-Cu2-N21 94.66(16).

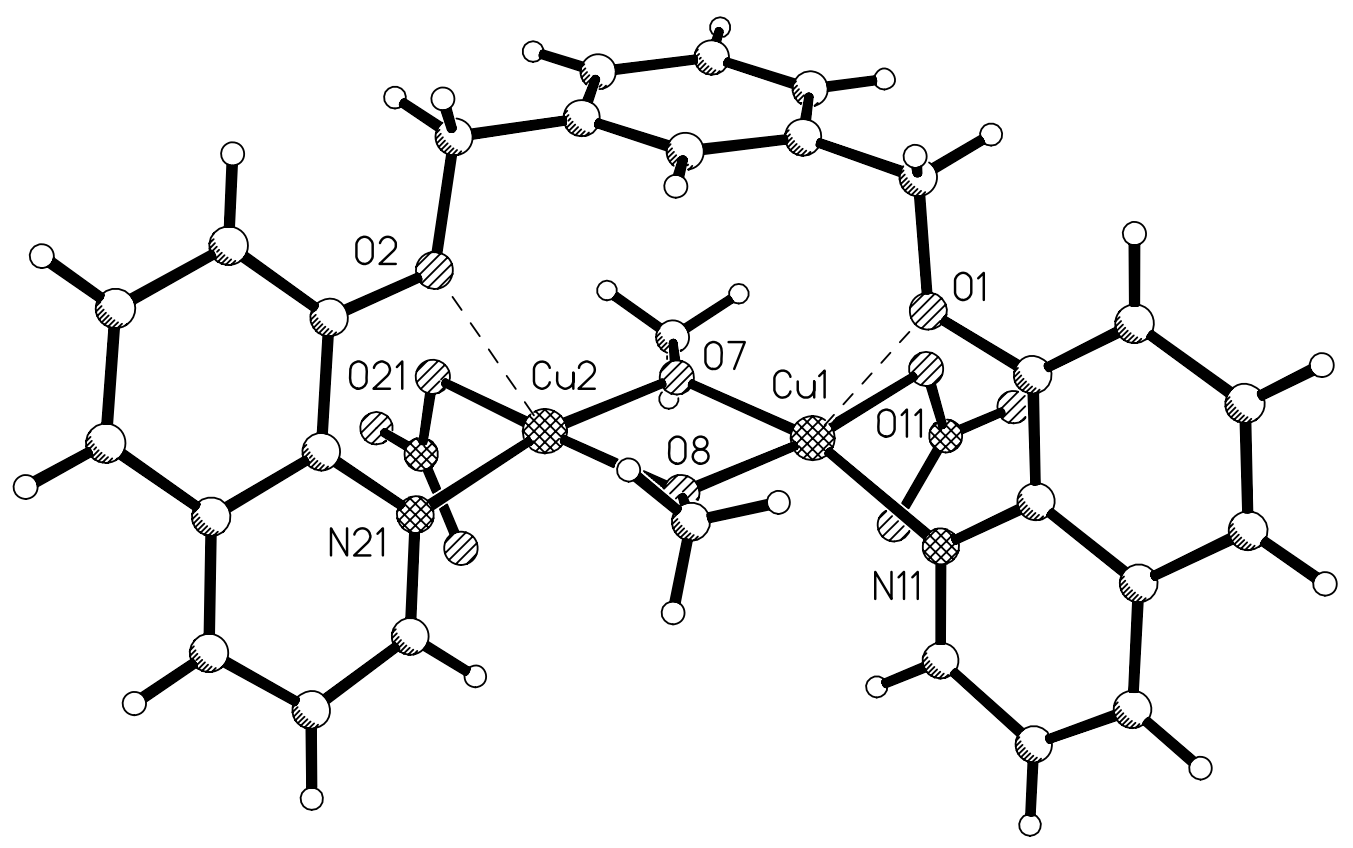


Fig. 6 Perspective view of the X-ray crystal structure of 10. Diethyl ether and acetonitrile solvate molecules are not shown. Selected interatomic distances $(\AA)$ and angles $\left({ }^{\circ}\right)$ :Cu1-O8 1.935(2), Cu1-N21 1.985(2), Cu1-O43 1.987(2), Cu1-O31 1.990(2), Cu1-O2 2.391(2), Cu2O7 1.937(2), Cu2-N11 1.975(2), Cu2-O11 1.987(2), Cu2-O21 2.010(2), Cu2-O1 2.395(2), O8-Cu1-N21 177.78(9), O8-Cu1-O43 87.87(9), N21-Cu1-O43 91.59(8), O8-Cu1-O31 86.74(9), N21-Cu1-O31 93.96(8), O43-Cu1-O31 172.91(7), O8-Cu1-O2 107.48(9), N21Cu1-O2 74.68(7), O43-Cu1-O2 90.45(7), O31-Cu1-O2 86.78(7), O7-Cu2-N11 175.81(10), O7-Cu2-O11 88.90(9), N11-Cu2-O11 94.01(9), O7-Cu2-O21 86.22(9), N11-Cu2-O21 90.98(9), O11-Cu2-O21 174.68(8), O7-Cu2-O1 102.09(9), N11-Cu2-O1 74.76(8), O11-Cu2O1 93.34(8), O21-Cu2-O1 89.74(8).

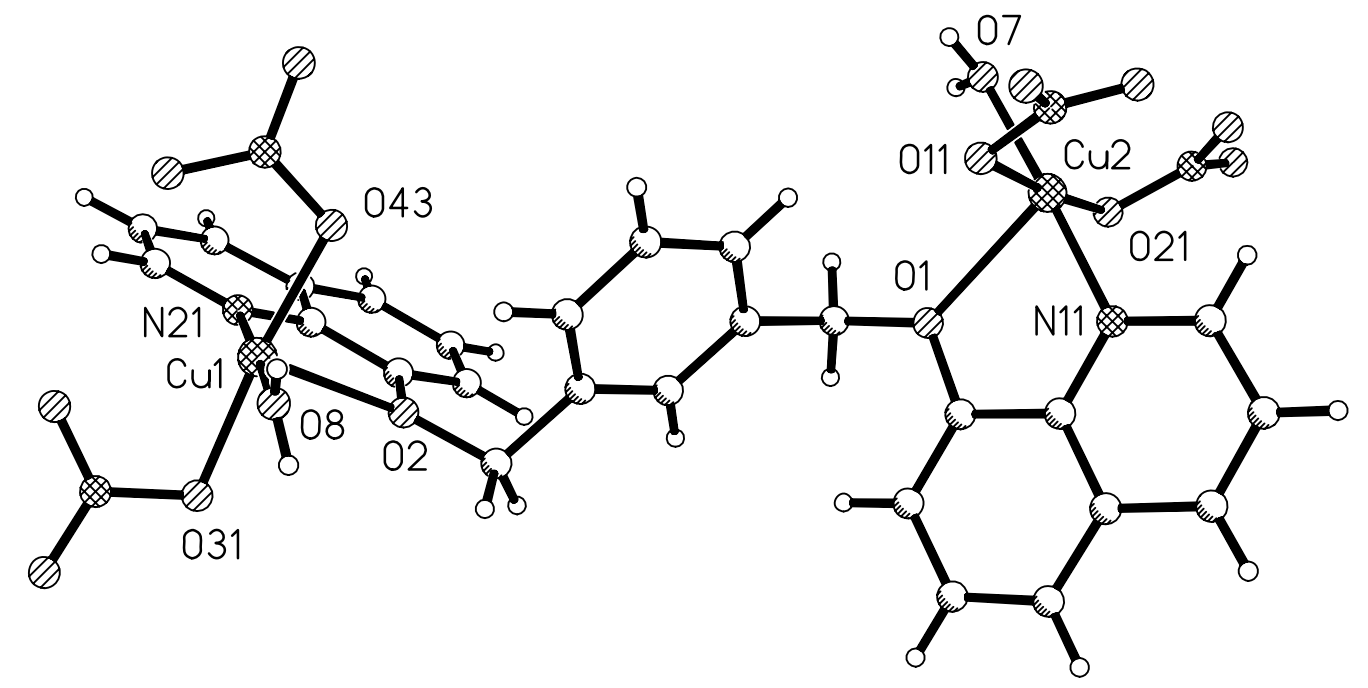


Table 1. Crystal Data and X-ray Experimental Details for 5-10.

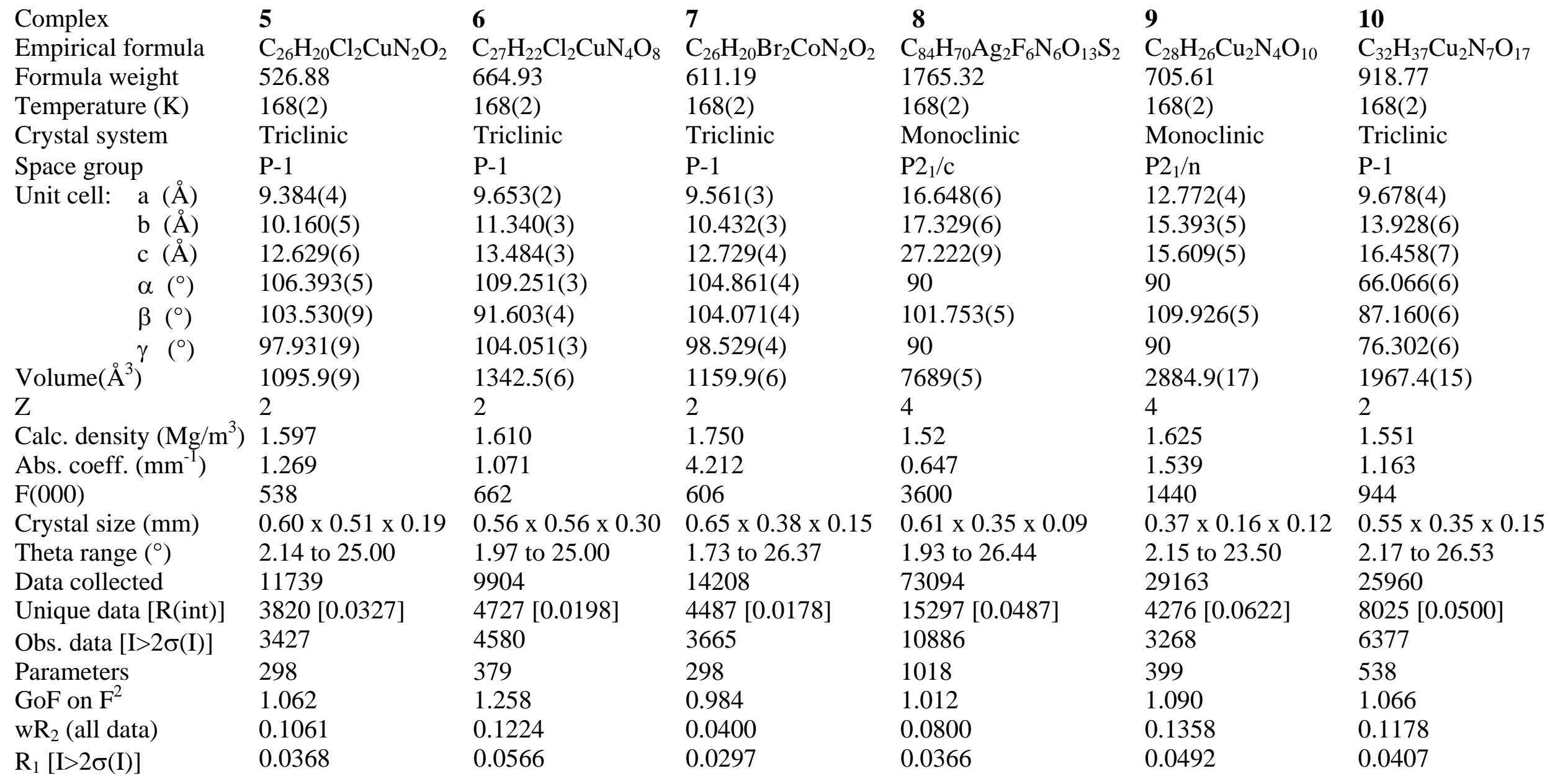




\section{Graphical Abstract}

\section{Muna R. A. Al-Mandhary, Peter J. Steel*}

X-ray crystallographic study of copper(II), cobalt(II) and silver(I) complexes of ligands containing two oxine subunits linked by xylylene spacer groups.

Keywords: N,O ligands / Copper / Cobalt / Silver / Bridging ligands

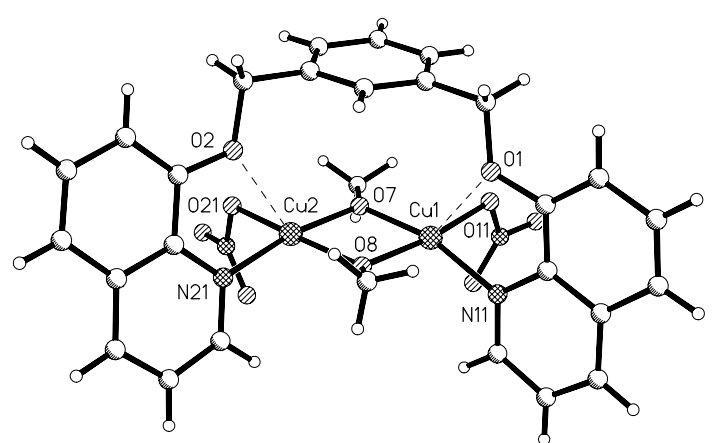

